\title{
Can unlicensed bands be used by unlicensed usage?
}

\author{
Liu Cui \\ lic49@pitt.edu \\ Martin BH Weiss \\ mbw@pitt.edu \\ School of Information Sciences \\ University of Pittsburgh \\ Pittsburgh PA 15260
}

\section{Introduction}

Since their introduction, unlicensed ISM bands have resulted in a wide range of new wireless devices and services. It is fair to say that the success of ISM was an important factor in the opening of the TV white space for unlicensed access. Further bands (e.g., 3550-3650 MHz) are being studied to support unlicensed access. Expansion of the unlicensed bands may well address one of the principle disadvantages of unlicensed (variable quality of service) which could result in a vibrant new group companies providing innovative services and better prices. However, given that many commercial mobile telephone operators are relying heavily on the unlicensed bands to manage growth in data traffic through the "offloading" strategy, the promise of these bands may be more limited than might otherwise be expected (Musey, 2013).

Wireless data traffic has exploded in the past several years due to more capable devices and faster network technologies. While there is some debate on the trajectory of data growth, some notable reports include AT\&T, which reported data growth of over 5000\% from 2008 to 2010 and Cisco, who predicted that mobile data traffic will grow to 6.3 exabytes per month in average by 2015 (Hu, 2012). Although the data traffic increased dramatically, relatively little new spectrum for mobile operators has come online in the last several years; further, the "flat-rate" pricing strategy has led to declining Average Revenue Per User (ARPU) for the mobile operators. Their challenge, then, is how to satisfy the service demand with acceptable additional expenditures on infrastructure and spectrum utilization.

A common response to this challenge has been to offload data traffic onto unlicensed (usually WiFi) networks. This can be accomplished either by establishing infrastructure (WiFi hotspots) or to use existing private networks. This phenomenon leads to two potential risks for spectrum entrants: (1) the use of offloading may overwhelm unlicensed spectrum and leave little access opportunities for newcomers; (2) the intensity of the traffic may increase interference and degrade innovative services.

Consequently, opening more unlicensed frequency bands alone may not necessarily lead to more unlicensed usage. In this paper, we will estimate spectrum that left for unlicensed usage and analyze risks for unlicensed users in unlicensed bands in terms of access opportunities and monetary gain. We will further provide recommendations that help foster unlicensed usage in unlicensed bands. 


\section{Related issues}

In this section, we will review two related issued of unlicensed usage in unlicensed bands. The first one is regulations in unlicensed bands, which includes regulations of Industrial, Scientific and Medical bands and TV white space (TVWS). The second topic reviews mobile offloading, which includes different types of mobile offloading, techniques that have been proposed for offloading, and business analysis for mobile offloading.

\subsection{Regulations in unlicensed bands}

\subsubsection{ISM}

The Industrial, Scientific and Medical (ISM) bands are frequencies that are reserved for industrial, scientific, and medical purposes other than communications ${ }^{1}$. They include the frequency bands $902-$ $928 \mathrm{MHz}, 2400-2483.5 \mathrm{MHz}$, and 5725-5850 MHz. Spectrum entrants with frequency hopping and direct sequence spread spectrum transmitters are permitted to operate with many requirements, including minimum separate of hopping channels, average time of occupancy on any frequency, and minimum hopping frequencies. Regulations that are related to transmission power are summarized here ${ }^{2}$ : Maximum transmitter output power fed into the antenna is $30 \mathrm{dBm}$ ( $1 \mathrm{Watt}$ ). Maximum EIRP is $36 \mathrm{dBm}$ (4 watt). Two exceptions for fixed point-to-point link: (1) there is no requirement on antenna gain in the $5.8 \mathrm{GHz}$; (2) in $2.4 \mathrm{GHz}$, system can increase antenna gain above $36 \mathrm{dBm}$ but for every $3 \mathrm{dBi}$ increase of antenna gain, the system have to reduce the transmit power by $1 \mathrm{dBm}$.

WiFi and Bluetooth are two dominant protocols for communications in ISM bands. Although sensing is not required for unlicensed devices in ISM bands, IEEE 802.11 mandates devices to sense the carrier before operation in order to avoid interference.

\subsubsection{TVWS}

Becons, Geo-location database, and sensing are three major ways to access TVWS. With beacon methods, unlicensed devices are allowed to transmit only if the control signal identifies vacant channels within their coverage. In Geo-location database, unlicensed devices must be equipped with a GPS receiver to determine its location and then check the TVWS database to find vacant TV channels at their locations. In the sensing method, unlicensed devices detect primary users' signals and only transmit when primary users are absent (Nekovee, 2010).

Each method has unique problems. For example, beacon and geo-location database requires an entity to build the infrastructure and maintain it. The problem for beacon and sensing is that signals may experience the hidden node problem, which reduces the TVWS usage efficiency or may result in harm to the incumbent systems (Nekovee, 2010).

\footnotetext{
${ }^{1}$ ARTICLE 1 - Terms and Definitions" (HTML). life.itu.ch. International Telecommunication Union. 19 October, 2009. 1.15. "industrial, scientific and medical (ISM) applications (of radio frequency energy): Operation of equipment or appliances designed to generate and use locally radio frequency energy for industrial, scientific, medical, domestic or similar purposes, excluding applications in the field of telecommunications."

${ }^{2}$ Part 15, Subpart C, Sec. 15.247 Operation within the bands $902-928 \mathrm{MHz}, 2400-2483.5 \mathrm{MHz}$, and 5725-5850 MHz.
} 
In 2008, the FCC released the Second Report and Order (FCC, 2008) to allow unlicensed devices to transmit in the broadcast television spectrum at locations where licensed services are absent. These unused TV channels are referred to as white spaces. In this document, the FCC requires:

- All devices (except personal/portable devices operating in client mode), must have geo-location capability and must access the database to obtain a list of the permitted channels before transmission. They also must have a capability to sense TV broadcasting and wireless microphone signals, at levels as low as $-114 \mathrm{dBm}$.

- Fixed devices can operate on any channel between 2 and 51, except channels 3, 4, and 37 (that is frequency bands $54-60 \mathrm{MHz}, 76-88 \mathrm{MHz}, 174-216 \mathrm{MHz}, 470-608 \mathrm{MHz}, 614-698 \mathrm{MHz}$ ), up to 4 Watts effective isotropic radiated power (EIRP). Similarly, personal portable devices can operate on channels between 21 and 51, except channel 37, which are the frequency bands of 512-608 $\mathrm{MHz}, 614-698 \mathrm{MHz}$. The transmission power cap in adjacent channels is 40 milliwatts, and for other channels is 100 milliwatts. Devices that only rely on sensing and do not have geolocation and database access capabilities are allowable, but they subject to a much more rigorous set of tests and the maximum transmission power is 50 milliwatts instead of 100 milliwatts. All devices must limit out-of-band emissions in the first adjacent channel to a level $55 \mathrm{~dB}$ below the power level in the channel they occupy.

- All devices must provide identifying information to the database for the sake of enforcement. All devices must have adaptable power control in order to use the minimum power to complete communications. All devices are subject to equipment certification by the FCC Laboratory before implementation.

In 2010, the FCC released the final rules for unlicensed usage in TV white space in the Second Memorandum Opinion and Order (FCC, 2010). The significant change in that document for unlicensed users is that the FCC eliminating the sensing requirement for TV bands devices with geo-location capability and ability to access the database. Second, the required in-band emission will be measured within $6 \mathrm{MHz}$ instead of $100 \mathrm{kHz}$. It also revised the attenuation level from $55 \mathrm{~dB}$ to $72.8 \mathrm{~dB}$. They require devices to re-check the database at least once daily after operation. Further, fixed device are permitted to transmit up to 1 watt in power and may use an antenna that provides up to $6 \mathrm{dBi}$ of gain.

\begin{tabular}{|l|l|l|l|}
\hline Types of TV bands devices & $\begin{array}{l}\text { Power limit (6 } \\
\mathrm{MHz})\end{array}$ & $\begin{array}{l}\text { PSD limit (100 } \\
\mathrm{kHz})\end{array}$ & $\begin{array}{l}\text { Adjacent channel limit (100 } \\
\mathrm{kHZ})\end{array}$ \\
\hline Fixed & $30 \mathrm{dBm}(1 \mathrm{Watt})$ & $12.6 \mathrm{dBm}$ & $-42.8 \mathrm{dBm}$ \\
\hline $\begin{array}{l}\text { Personal/portable (adj. } \\
\text { channel) }\end{array}$ & $16 \mathrm{dBm}(40 \mathrm{dW})$ & $-1.4 \mathrm{dBm}$ & $-56.8 \mathrm{dBm}$ \\
\hline Sensing only & $17 \mathrm{dBm}(50 \mathrm{~mW})$ & $-0.4 \mathrm{dBm}$ & $-55.8 \mathrm{dBm}$ \\
\hline All other personal/portable & $20 \mathrm{dBm}(100 \mathrm{~mW})$ & $2.6 \mathrm{dBm}$ & $-52.8 \mathrm{dBm}$ \\
\hline
\end{tabular}

Table 1. Maximum permissible power spectral density (PSD) for different TV bands devices

In 2012, the FCC further adjusted rules in Third Memorandum Opinion and Order (FCC, 2012). They defined fixed adjacent channel emission limit as the maximum power permitted in a $6 \mathrm{MHz}$ bandwidth 
minus $72.8 \mathrm{~dB}$. It also slightly increases the maximum permissible power spectral density (PSD) as described in table 1.

\subsection{Literature review on cellular traffic offloading}

\subsubsection{Different types of cellular traffic offloading}

The essence of cellular traffic offloading, also called mobile offloading, is to use complementary network technologies to transmit mobile data traffic that originally delivered through cellular networks. It is regarded as a win-win solution for cellular network operators and end-users. On one hand, cellular operators may provide better experience for end-users by alleviating the data traffic on the cellular network. Moreover, they may save the cost for updating infrastructures that required by the bursty data traffic. On the other hand, end-users have the opportunity to reduce their data cost, longer the battery life, and enhance the data rate (Han 2011).

In terms of frequency bands and scope, there are two types of cellular traffic offloading: femtocells for indoor offloading and WiFi for outdoor offloading. Femtocells work on the licensed bands as the macrocell. It provides higher capacity and better coverage for indoor communications. The major disadvantage of femtocell includes the need to deploy short-range base stations and this transmission may also interfere with data on macrocells, since they operate on the same frequency. On the contrary, WiFi offloading works on the unlicensed spectrum and has no impact on existing cellular networks. However, WiFi offloading may experience higher interference from other unlicensed devices (Han 2011, Dimatteo, 2011).

Considering allowable traffic delay, cellular traffic offloading can be divided into two categories: on-thespot and delayed. On-the-spot offloading use spontaneous connectivity to WiFi and offload data on the spot. Whenever users disconnect from WiFi, all the unfinished data will be transmitted through cellular networks. On the other hand, in delayed offloading mode, each data transfer is associated with a deadline. Data will be transmitted through WiFi network primarily, and the cellular networks will take over the transmission when data transfer does not finish within the deadline. On-the-spot offloading has been adopted by most mobile providers (Lee, 2010).

\subsubsection{Cellular traffic offloading schemes and business analysis}

Developing cellular traffic offloading schemes is a rising issue in research topic due to the potential benefits for mobile operators. There are two major directions of cellular traffic offloading schemes: delayed offloading and opportunistic-communication based offloading. Delayed offloading is opposed to instantaneous transmission, which means end-users can tolerate a certain period time for receiving the data. In opportunistic-communication based offloading, mobile operators first send information via cellular networks to users in a target set. Then, they rely on these users to propagate information among all end users through opportunistic communications. Finally, mobile operators will send information to end users who cannot receive it before the delay-tolerance threshold (Han 2011).

The authors of (Han 2011) focus on how to choose a target set in opportunistic-communication based offloading in order to maximize the reduction of mobile data traffic. They applied three algorithms: random, greedy, and heuristic. Their study targets on mobile offloading in metropolitan areas. Delivery 
efficiency can be improved by identifying the social networks of users and then delivering specific information to a particular social group (Hui, 2011). In another approach, delay tolerant networks are used to offload bulk file transfer and video streaming on both uplinks and downlinks (Dimatteo, 2011). They quantify the number of needed Access Points (APs) for offloading data with different quality of service (QoS) requirements in a large metropolitan area.

Traffic offloading can also be accomplished by a self-organizing process (Simsek, 2012). Instead of offloading as much data as possible, this procedure allows small cells to learn their optimal transmission configuration over both licensed and unlicensed bands and then decide which band to use. In this way, small cells may use the licensed band when WiFi is congested and vice versa.

Offloading has also been examined from a business standpoint (GrøNsund, 2012) as well. This paper compares traditional femtocell offloading with sensor network aided offloading. With sensor network ancillary, a femtocell will be able to use frequencies other than licensed bands. In this way, both coverage and capacity may be improved. The comparison is based on the potential cost savings and sensitive analysis.

\section{Why it is an issue}

Due to the success of unlicensed bands in accommodating a variety of services, opening up more unlicensed bands have been an emerging issue. By doing that, the regulator aims at enhancing development of novel applications, service models, and technologies. However, increasing the quantity of unlicensed bands does not necessarily bring more unlicensed usage due to mobile offloading and other potential incumbents' usage. Unlicensed users have certain QoS requirements; if the unlicensed bands cannot satisfy their requirements, they won't establish infrastructure there. From the perspective of unlicensed frequency usage, there are many factors that impact the quantity and quality of unlicensed bands. Therefore, before regulators draw the conclusion that more unlicensed frequency will bring more unlicensed usage, they have to quantify the capacity that is available for unlicensed usage.

There is some research on quantifying capacity in unlicensed bands. However, most of it focused on calculating and measuring number of available channels. For example, (Van De Beek, 2011) found out that $56 \%$ of the TV channels are unused in Europe, when averaged over the whole geographic area. If this number is averaged over population, $49 \%$ of the TV channel is available for unlicensed usage. (Snider, 2007) estimates the average amount of white-space per person is $214 \mathrm{MHz}$. This number is based on the FCC's estimation that "American can receive 13.3 channels and there are 49 total DTV channels of $6 \mathrm{MHz}$ each". In (Mishra, 2010), the authors estimate how much spectrum-measured in the number of channels - the FCC rules open up based on the 2000 USA census and TV tower data extracted from the FCC database. These research works tend to overestimate the capacity, since they do not consider interference from primary users, interference among secondary users, regulations, and cellular traffic offloading.

(Dudda, 2012) and (Harrison, 2010) improves above research by considering regulations and interference from other unlicensed devices. (Dudda, 2012) quantifies TV white space in Germany by 
using the European methodology developed in CEPT ECC SE 43. They consider the interference from TV towers and limited transmitting power cap for unlicensed devices work in TV white space. (Harrison, 2010) provides a realistic evaluation of TV white space capacity for United States. They consider the impact of wireless pollution from existing television stations, the self-interference among whitespace devices themselves, the population distribution, and the expected transmission range of the whitespace devices.

Although (Dudda, 2012) and (Harrison, 2010) made more realistic estimates for TVWS with concerns on regulations and interferences, they do not consider traffic offload from mobile operators. In the meantime, mobile traffic offload is a large part of unlicensed usage. (Lee, 2010) measures WiFi offload usage and concludes that $65 \%$ of the total mobile data traffic is transmitted on WiFi networks now. Hence, in this paper we will estimate the capacity that left for unlicensed usage and access the risks that unlicensed users may face.

\section{What is left for unlicensed users?}

When calculating capacity and probability of blocking, we divide the unlicensed spectrum into ISM and TVWS. In the ISM band, there is no primary user; whereas in TVWS, the TV stations and microphones are primary users. We only consider TV stations as primary users in this analysis. We quantify capacity as time-varying behavior, and adopt a Markov model. In this paper, we consider the simple scenario in which all secondary users can perfectly detect each other's usage, although they do not need to cooperate with each other. Moreover, they can also perfectly detect primary usage. In future research, we will develop this model into multi-dimension Markov model that considers interference among users and impact from spectrum users' locations (eg., propagation effects and sensing capabilities).

\subsection{Time-varying Markov Model}

\subsubsection{Capacity left for unlicensed users in ISM bands}

Following (Patil, 2010) the state transition diagram of the scenario without primary users is depicted in Figure 1. It is assumed that the ISM band can accommodate at most c users. In this model, state 0 (idle) means there are no users, and state $S U_{i}$ represents that there are $i$ users in the channel. Eq.(1) is the associated generator matrix for this Markov Process. $\lambda_{s}$ and $\mu_{s}$ is the average arrival and holding time, respectively.

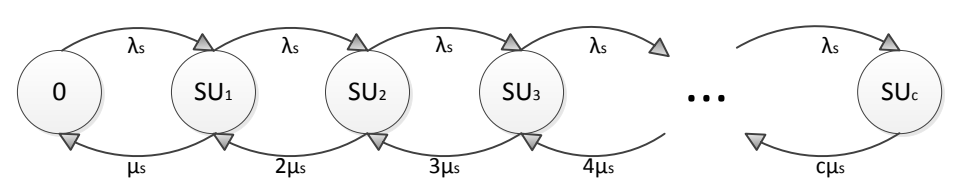

Figure 1. State transition diagram of scenario without primary users 


$$
Q_{1}=\left(\begin{array}{ccccc}
-\lambda_{s} & \lambda_{s} & 0 & \cdots & 0 \\
\mu_{s} & -\left(\mu_{s}+\lambda_{s}\right) & \lambda_{s} & \cdots & 0 \\
0 & 2 \mu_{s} & -\left(2 \mu_{s}+\lambda_{s}\right) & \cdots & 0 \\
0 & 0 & 3 \mu_{s} & \cdots & 0 \\
\vdots & \vdots & \vdots & \ddots & \vdots \\
0 & 0 & 0 & \cdots & -c \mu_{s}
\end{array}\right)
$$

As stated above, we regard spectrum access as a time varying process. Therefore, we let $\lambda_{s}(t)$ and $\mu_{s}(t)$ represent the mean arrival and holding time, respectively. $p_{j}(t)$ is the probability of $j$ users being in the system at time $t$. For nonstationary finite state Markovian processes, the time dependent behavior can be determined numerically by integrating the associated Chapman-Kolmogorov differential equation model (Tipper, 1990). The Chapman-Kolmogorov differential equations for the time varying state probabilities of the model represented by Figure 1 are given by,

$$
\begin{aligned}
& \frac{d p_{0}(t)}{d t}=-\lambda_{s}(t) p_{0}(t)+\mu_{s} p_{1}(t) \\
& \frac{d p_{j}(t)}{d t}=-\lambda_{s}(t) p_{j-1}(t)-\left(\lambda_{s}(t)+j \mu_{s}\right) p_{j}(t)+(j+1) \mu_{s} p_{j+1}(t) \quad(1 \leq j<c) \\
& \frac{d p_{c}(t)}{d t}=-\lambda_{s}(t) p_{c-1}(t)+c \mu_{s} p_{c}(t)
\end{aligned}
$$

This differential equation model can be solved numerically using the following steps. First, determine start $\left(t_{0}\right)$ and finish time $\left(t_{f}\right)$; set current time $t$ to $t=t_{0}$, establish the initial system state probability vector and specify a time step $\delta_{t}$. Next, approximate the arrival rate by a constant over $\delta_{t}$ (e.g., $\lambda_{s}=\lambda_{s}\left(t+\delta_{t} / 2\right)$. Third, apply a standard numerical integration algorithm to solve the differential equation. Fourth, increase time, $t=t+\delta_{t}$, if $t<t_{f}$, go to step 2, else stop. Using this model, we calculate the probability of blocking $\left(P_{b}\right)$ as $P_{b}=P_{c}$.

\subsubsection{Capacity left for unlicensed users in TVWS}

In TVWS, we only consider TV stations as primary users. The state transition diagram with primary usage is depicted in Figure 2, where $\lambda_{p}$ and $\mu_{p}$ represent the mean arrival and holding time for primary usage, respectively. The state PU describes the situation where primary usage is present. The primary user has the priority to occupy the channel in a non-preemptive fashion. After the primary user leaves, the system model will first return to the idle state and then secondary users start to access the spectrum. As regulated by the FCC, secondary users can determine primary usage by geolocation database and sensing. 


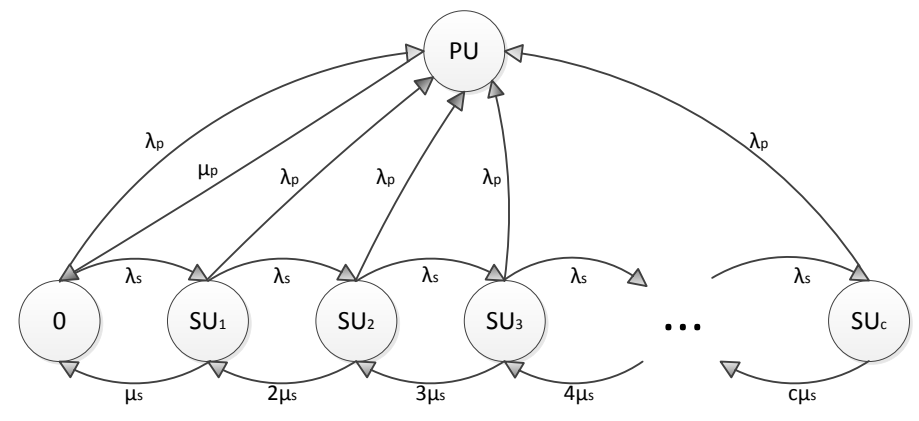

Figure 2. State transition diagram with primary usage

The generator matrix of the Markov Process is described in Eq. (3),

$$
Q_{2}=\left(\begin{array}{ccccccc}
-\mu_{p} & \mu_{p} & 0 & 0 & \cdots & 0 & 0 \\
\lambda_{p} & -\left(\lambda_{p}+\lambda_{s}\right) & \lambda_{s} & 0 & \cdots & 0 & 0 \\
\lambda_{p} & \mu_{s} & -\left(\lambda_{p}+\mu_{s}+\lambda_{s}\right) & \lambda_{s} & \cdots & 0 & 0 \\
\lambda_{p} & 0 & 2 \mu_{s} & -\left(\lambda_{p}+2 \mu_{s}+\lambda_{s}\right) & \cdots & 0 & 0 \\
\lambda_{p} & 0 & 0 & 3 \mu_{s} & \cdots & 0 & 0 \\
\vdots & \vdots & \vdots & \vdots & \ddots & \vdots & \vdots \\
\lambda_{p} & 0 & 0 & 0 & \cdots & -\left(\lambda_{p}+(c-1) \mu_{s}+\lambda_{s}\right) & \lambda_{s} \\
\lambda_{p} & 0 & 0 & 0 & \cdots & c \mu_{s} & -\left(\lambda_{p}+c \mu_{s}\right)
\end{array}\right) \text { (3) }
$$

In the time-varying model, we assume the arrival process for primary and secondary users are $\lambda_{p}(t)$ and $\lambda_{s}(t)$, respectively. The Chapman-Kolmogorov differential equations are listed in Eq. (4). The procedures for solving this model are the same as above.

$$
\begin{aligned}
& \frac{d p_{p u}(t)}{d t}=\lambda_{p}(t) \sum_{i=1}^{c} p_{i}(t)+\mu_{p} p_{p u}(t) \\
& \frac{d p_{0}(t)}{d t}=-\left(\lambda_{p}(t)+\lambda_{s}(t)\right) p_{0}(t)+\mu_{p} p_{p u}(t)+\mu_{s} p_{1}(t) \\
& \frac{d p_{j}(t)}{d t}=\lambda_{s}(t) p_{j-1}(t)+(j+1) \mu_{s} p_{j+1}(t)-\left(\lambda_{s}(t)+j \mu_{s}+\lambda_{p}(t)\right) p_{j}(t) \quad(1 \leq j<c) \\
& \frac{d p_{c}(t)}{d t}=\lambda_{s}(t) p_{c-1}(t)-c \mu_{s}+\lambda_{p}(t) p_{c}(t)
\end{aligned}
$$

\subsection{Traffic model}

As mentioned by (Lee, 2010), Wifi already offloads about $65 \%$ of the total mobile data traffic. (Informa, 2013) further pointed out that WiFi accounted for $73 \%$ of total traffic on Android smartphones in April, up from 67\% in August 2013. The average total cellular data usage for US Android smartphone users is $1,250 \mathrm{MB}$ per month. Although we have actual usage measurement on mobile offload data size, we do not know the average arrival rate and holding time for WiFi offloading. The mobile offloading traffic model that described below is modified from (Willkomm, 2010), which measures cellular network usage and provided average arrival rate and holding time for voice communication. Although that is different from data usage, it provides a baseline for the cellular network traffic shape. With more accurate arrival 
and holding time, we will be able to provide more precise statistics on spectrum access, such as the blocking rate.

In this analysis, we model three types of traffic for mobile offloading. The reason behind the first traffic model is that WiFi offload tends to be more intense during the day time. Urban area is an example for this offload model. The second offload model shows that the peak offload comes during the night time. Locations such as shopping malls may have this characteristic. By comparing these two models, we will discover the impact with respect to time. The third traffic model is time invariant.

There are also two traffic models for unlicensed usage (indicated by SUx in figure 3). The first unlicensed usage has similar shape as traffic offloading. That means the usage is high during the day time. This is the case for human related applications. The second unlicensed usage has more usage before dawn. Machine-to-machine communications which send out reports or daily updates during night time is an example for this traffic model.

Primary usage is modeled as "on-off" process in this paper, since traffic from TV stations is predictable.

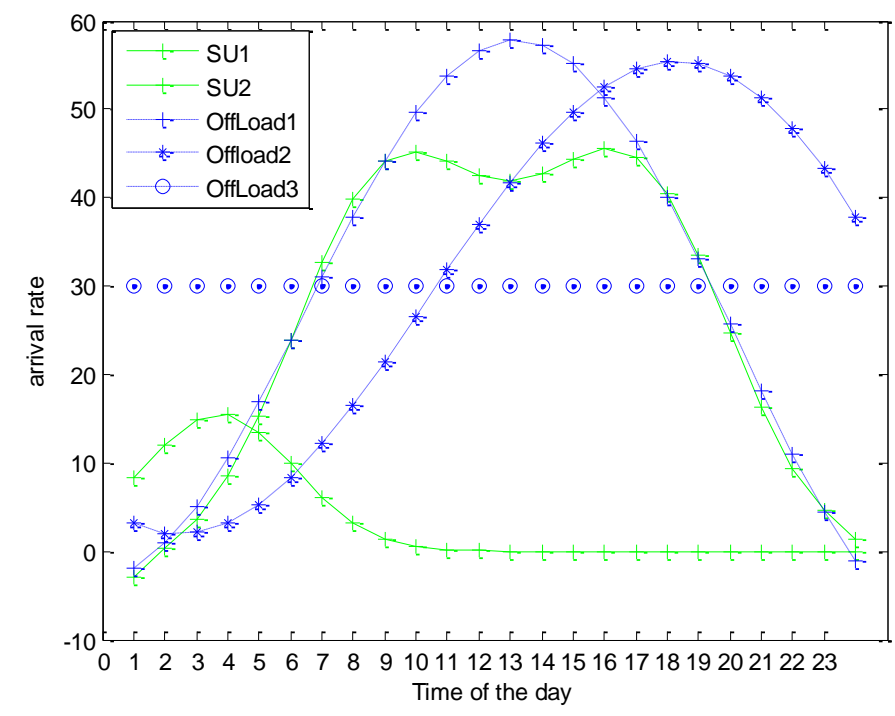

Figure 3. Traffic models for offloading and unlicensed usage

\subsection{Numerical Results}

In this section, numerical results of estimating probabilities of blocking in different scenarios are provided.

\subsubsection{Blocking rate from different offload traffic model in ISM bands}

In this section, it is assumed that there is no primary usage. We calculate the probability of blocking in two cases. In the first case, the ISM bands can accommodate at most six users $(c=6)$, as shown in Figure 4. In the second case, we double this number, which means ISM can accommodate at most twelve users $(c=12)$, as shown in Figure 5. The primary reason for using these two cases is that unlicensed bands have different bandwidth, and therefore support different numbers of users. For 
example, the ISM band in range $2.4 \mathrm{GHz}$ to $2.4835 \mathrm{GHz}$ has bandwidth of $83.5 \mathrm{MHz}$, while TVWS has bandwidth of $6 \mathrm{MHz}$ (per channel). The second reason is that service type also impacts the number of users that can be accommodated in the same band. For example, machine-to-machine communications requires less resource, while large data and voice traffic needs to occupy a larger bandwidth. Within each case, we test all three different mobile offloading traffic models.
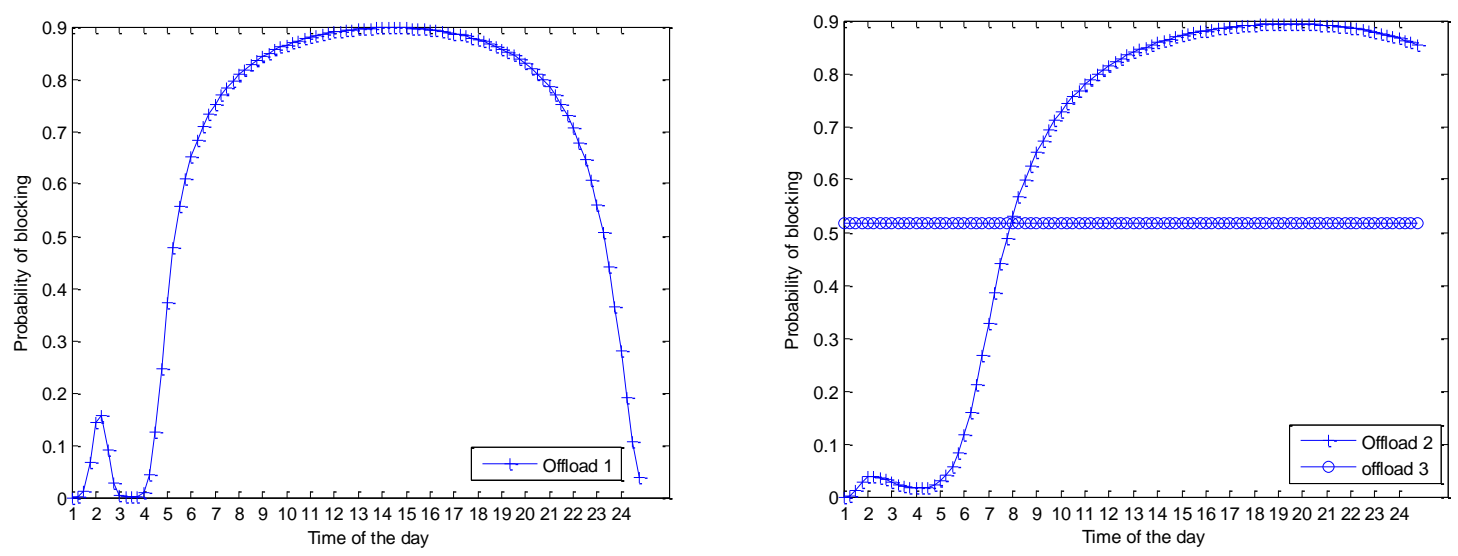

Figure 4. (a) Probability of blocking for offload traffic model 1 without primary usage and c=6.

(b) Probability of blocking for offload traffic model 2 and 3 without primary usage and c=6.

Not surprisingly, the more users that ISM bands can accommodate, the lower probability of blocking. Moreover, with different mobile offloading traffic, the probability of blocking varies with time. For example, the peak of probability of blocking for Offloading Traffic 1 that has a large daytime usage is between 8 am to $7 \mathrm{pm}$. The peak of probability of blocking for Offloading Traffic 2 shifts to $4 \mathrm{pm}$ to $12 \mathrm{am}$.
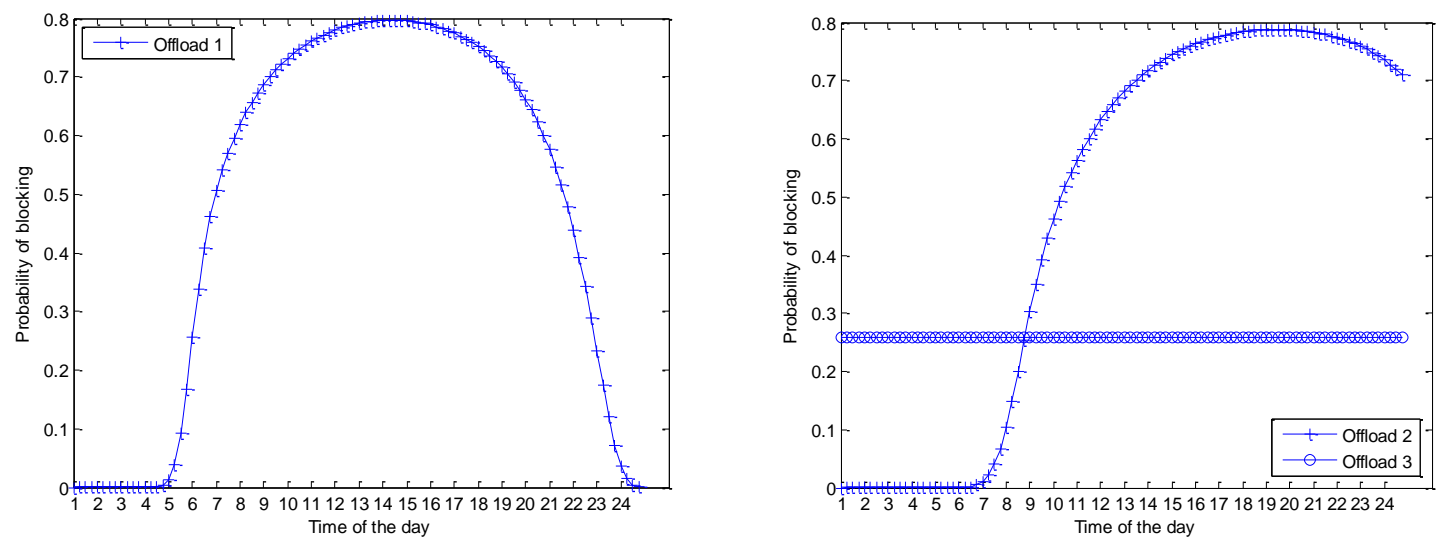

Figure 5. (a) Probability of blocking for offload traffic model 1 without primary usage and c=12.

(b) Probability of blocking for offload traffic model 2 and 3 without primary usage and c=12. 


\subsubsection{Blocking rate from different offload traffic model in TVWS}

We estimate the probability of blocking in TVWS with $c=6$ and $c=12$ as well. They show similar features as Figure 4 and 5, except when primary usage is present, in which case the probability of blocking becomes 1 . That means, no secondary usage is allowed in that region. This result may vary depending on the distance between TV stations and secondary users' operating regions. We will explore the impact from distance with multi-dimension Markov process in the future.
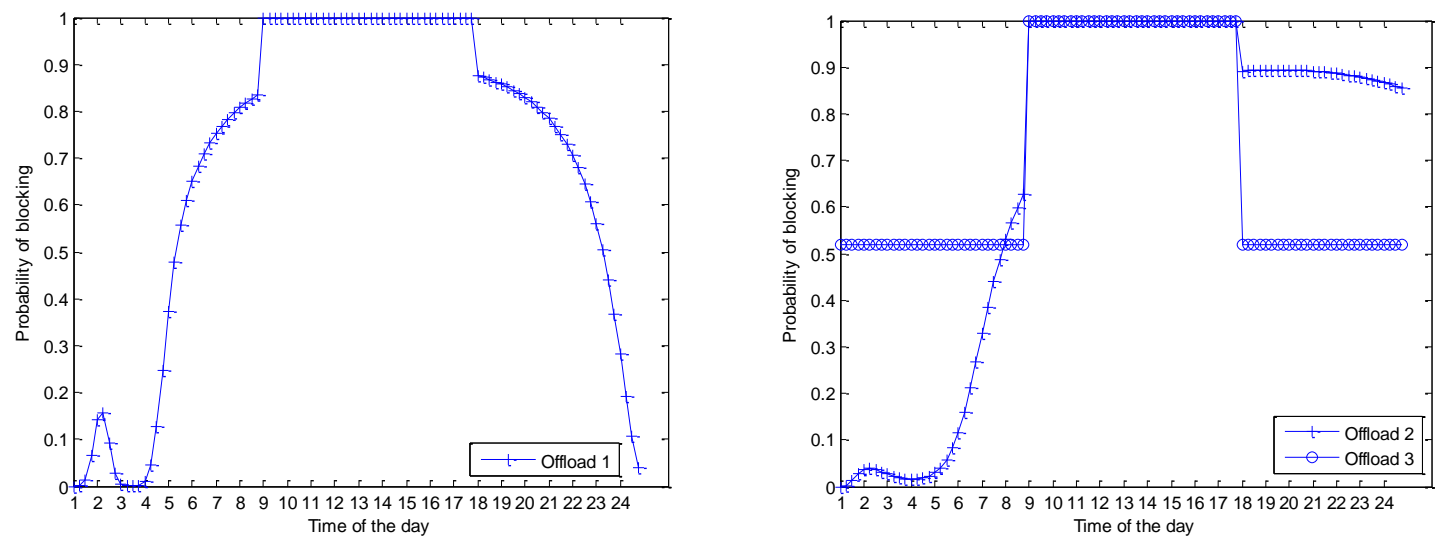

Figure 6. (a) Probability of blocking for offload traffic model 1 with primary usage and c=6.

(b) Probability of blocking for offload traffic model 2 and 3 with primary usage and c=6.
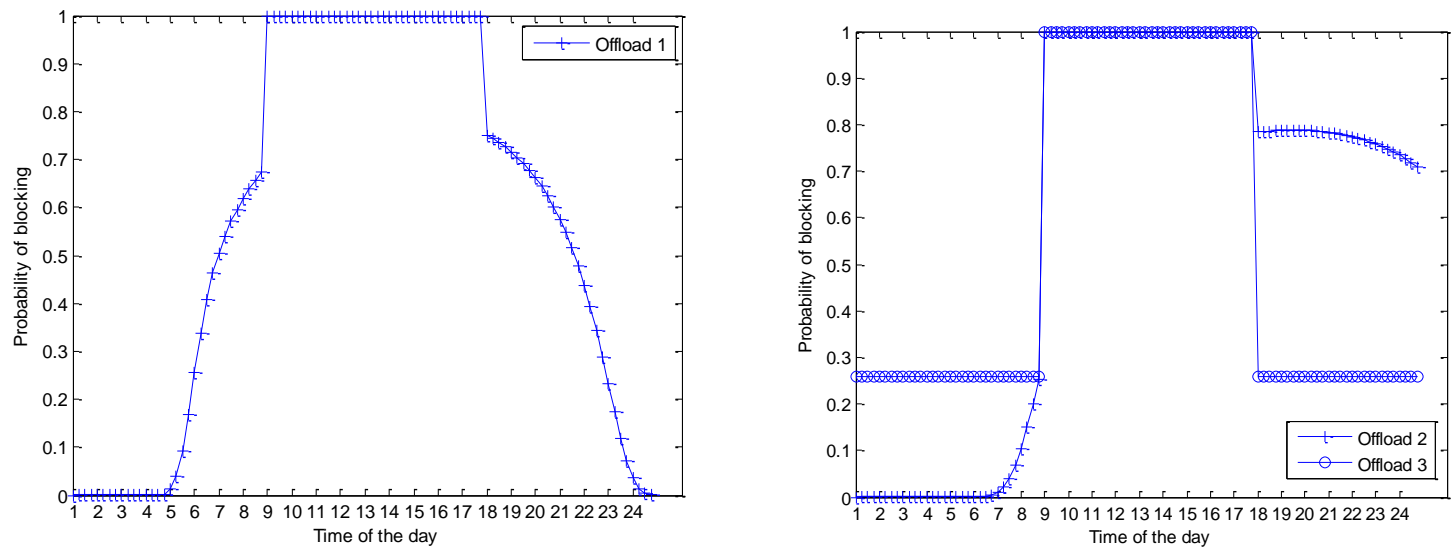

Figure 7. (a) Probability of blocking for offload traffic model 1 without primary usage and c=12.

(b) Probability of blocking for offload traffic model 2 and 3 without primary usage and c=12.

\section{Monetary risks for unlicensed users considering mobile traffic offload} In this section, we will estimate the monetary risks for unlicensed users brought by mobile traffic offloading. Cost and different revenue models are provided. In the numerical results, sensitivity analyses are included to show the impact from different factors. 


\subsection{Cost model}

There are two components in the unlicensed cost model: initial cost and recurrent cost. Initial cost includes base station cost $\left(C_{B}\right)$ and transmitter cost $\left(C_{T}\right)$. Recurrent cost includes backhaul cost $\left(C_{B h}\right)$, maintenance cost $\left(C_{M}\right)$, and research and business expenses $(\delta)$.

$$
\begin{gathered}
\text { Initial cost }=C_{B}+C_{T} \\
\text { Recurrent cost }=C_{B h}+C_{M}+\delta
\end{gathered}
$$

Unlicensed users have the least infrastructure cost per station because the equipment is heavily standardized and the transmit power is limited. It is assumed that the reach for WiFi transmitter is $100 \mathrm{~m}$ (outdoors). We assume that the cost for each base station is $\$ 600$ including transmitters $(\$ 500)$ and installation ( $\$ 100)$. The backhaul cost is assumed to be $\$ 50$ per month (similar to DSL) per site, although this could be reduced by careful attention to the access network architecture. It is further assumed that maintenance cost is $10 \%$ of the infrastructure (transmitter) cost.

We use two cases to illustrate our idea. In the first case, the unlicensed user want to provide large coverage, say $30,000,000 \mathrm{~m}^{2}$. Therefore, they need 950 base stations to cover the entire region. In the second case, the unlicensed users only provide short range services that require 1 base station.

\subsection{Revenue model}

In the revenue model, we consider the worst case where mobile offloading have higher priority and unlicensed usage can only operate on spared channel. We are aware that 802.11 uses "listen-before-talk" and all users in unlicensed bands have the same priority. There are two reasons for using a worst case analysis. First, mobile offloading normally contains large bulk data which may occupy the channel. Second, although "listen-before-talk" is in use, mobile offloading may interfere with unlicensed usage, which is hard to estimate. In this paper, we will use three revenue functions to estimate revenues for unlicensed users with sensitivity analysis.

In order to simplify the analysis, we only calculate monetary risks for offloading traffic model 1 with high blocking rate in ISM bands (Figure 5 (a)). Figure 8 shows the probability of blocking for unlicensed usage 1 and 2 (SU1 and SU2 in Figure 3) due to the impact from mobile offloading. As described above, unlicensed usage 1 is characterized by high access rates during the daytime and unlicensed usage 2 has heavy access before dawn.

The first type of revenue function that will be adopted in this analysis is linear with number of access. As state above, the probability of blocking is the worst case. Therefore, Number of access under offloading $=$ $(1-$ probability of blocking $) \times$ unlicensed users'number of access .

Thus, unlicensed users revenue $=C_{n a} \times$ number of access under of loading. Where, $C_{n a}$ is the unit cost per number of access.

Data size is another criterion for charging for service. However, it is difficult to estimate transmitted data size for each user. In this analysis, we will calculate two cases for each unlicensed usage scenario in 
order to complete sensitivity analysis. In the first case, we consider that the holding time for each user is long, while the holding time is low in the second case. It is reasonable that when holding time is long, the average number of access is low and vice versa.

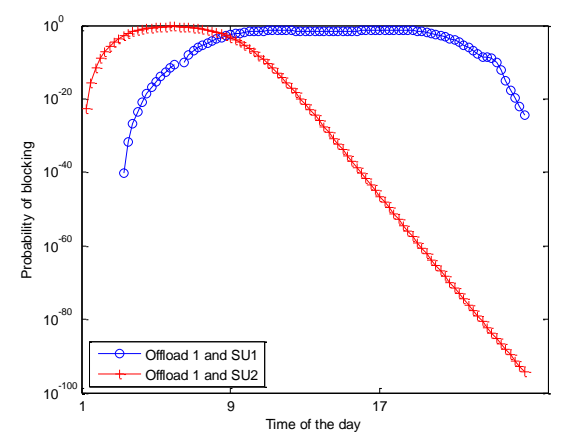

Figure 8. Probability of blocking for different unlicensed usage with short holding time

The step function that used by each unlicensed usage is different. First, we calculate the average data size for each user under different unlicensed usage based on our assumption. Then, the unit price is attached to calculate the revenue, as shown in Eq.(5). In long holding time, we assume that $20 \%$ of the accesses come from different users. In short holding time, we assume that $50 \%$ of the access come from different users.

$$
\begin{gathered}
P_{s 1}=\left\{\begin{array}{l}
1 \text { per user, average data size }<100000 \\
3 \text { per user, average data size }>100000
\end{array}\right. \\
P_{s 2}=\left\{\begin{array}{l}
3.5 \text { per user, average data size }<500000 \\
12 \text { per user, average data size }>500000
\end{array}\right.
\end{gathered}
$$

Where, $P$ is the step function. $a$ and $b$ are unit price, and $\varepsilon$ is the threshold for determining unit price.

The third type of revenue function increases with capacity linearly. In above two revenue functions, we consider the worst case scenario where mobile offloading will block unlicensed usage. It is possible that mobile offloading will not block unlicensed usage, but interfere with unlicensed usage. Therefore, we consider revenue increase with capacity linearly in this function. We adopt the transmission power level, 4 watts, which is determined by the FCC. Radius, interference level, and frequency bands are three important factors for capacity. Therefore, two levels of each factor are applied for the sensitivity analysis. In detail, unlicensed users revenue $=C_{c} \times$ capacity, where, $C_{c}$ is the unit cost per bps. Capacity will be determined by Shannon capacity formula, Capacity $=B \times \log _{2}(1+S / N)$, where, $B$ is the bandwidth in Hertz, $S$ and $N$ are signal and noise level in watts. Signal strength is calculated by free space propagation model, $F S P L=\left(\frac{4 \pi d f}{c}\right)^{2}$, where $d$ is the distance between transmitter and receiver, $f$ is the transmission frequency, and $c$ is the speed of light. 


\subsection{Numerical results}

\subsubsection{Assumptions}

It is assumed that unlicensed usage 1 (SU1) is used by people, and population density is not uniformly distributed. Therefore, we assume that only 500 base stations have high demand as described in Figure 3. The other 450 base stations experience low access rate. However, with machine-to-machine communications (Unlicensed Usage 2), we assume that the number of access is uniformly distributed throughout all base stations. Since small coverage only has one base station, the number of access is high as shown in Figure 3.

In the revenue function that linearly increases with capacity, we calculate NPV profits for $100 \mathrm{~m}$ radius in ISM $(2.5 \mathrm{GHz})$ and TVWS $(700 \mathrm{MHz})$ bands. The bandwidth for ISM and TVWS is $83.5 \mathrm{KHz}$ and $6 \mathrm{MHz}$, respectively. It is assumed that the low interference level (without mobile offloading) is 0.001 watt and high interference level (with mobile offloading) is 1 watt.

There are two coefficients that used to calculate revenues for the first revenue model, 0.0015 (Low) and 0.004 (High). The coefficient for the second model is listed in Eq.(5). The linear coefficient for capacity is assumed to be 0.1 per bit per second (bps).

\subsubsection{Numerical results and discussions}

In this section, we show numerical results for NPV profit that is calculated by

$$
N P V \text { Profit at year } n=\sum_{t=0}^{n} \frac{R_{t}-C_{t}}{(1+i)^{t}}
$$

Where, $R_{t}$ and $C_{t}$ are revenue and cost at year $t$, respectively. $R_{t}-C_{t}$ represents the net cash flow at year $t . i$ is the discount rate and is assumed to be 0.01 in this paper.

We will show the figure for NPV profits for revenue linearly increase with number of access in large coverage and use one table to compare NPV profits for all cases. Detailed tables can be found in Appendix. In Figure 9, "SUx" indicates traffic model for unlicensed usage. They are insisted with traffic model that are shown in Figure 3. " $\mathrm{H}$ " means the probability of blocking is high compared to situation where $c=12$. "OL" means mobile offloading is considered, while "NOL" means mobile offloading is neglected. Letter " $\mathrm{L}$ " and " $\mathrm{H}$ " represent the unit price for linear revenue functions. " $\mathrm{L}$ " means the unit price is 0.0015 , and " $H$ " means the unit price is 0.004 . We estimate each situation for a 10 year period. The horizontal axis is time (year 0 to year 10), and the vertical axis is the dollar value.

Table 3 summarizes NPV profits for all cases. The first part of the table illustrates the profits that unlicensed users can get by applying revenue functions that increase linearly with number of accesses. The second part of the table illustrates the profits that unlicensed users can get when the revenue is a step function that increases with data size. The difference between left and right side of Table 3 for these two cases is the coverage. On the left side, unlicensed users establish 950 base stations to cover $30,000,000 \mathrm{~m}^{2}$, while unlicensed users only establish one base station on the right side. The third part of the table analyzes NPV profits that unlicensed users can get if their revenue linearly increases with 
capacity. The first three columns here is NPV profits when the coverage radius is $100 \mathrm{~m}$, and the coverage radius for last three columns are $50 \mathrm{~m}$. All numbers in Table 3 are NPV profits at the $10^{\text {th }}$ year.

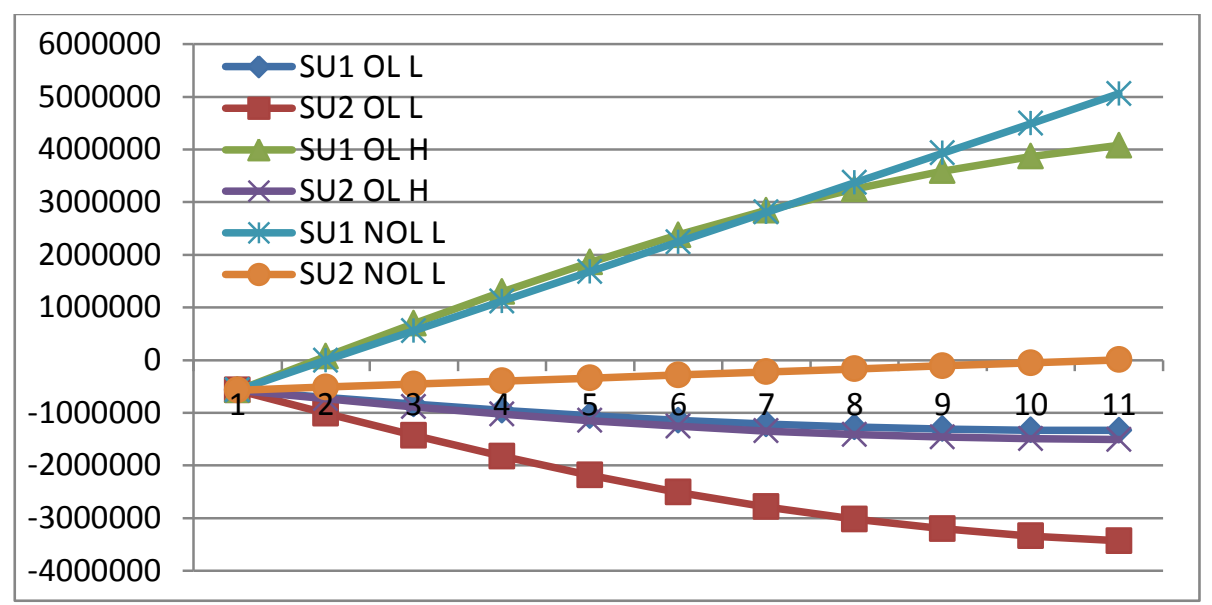

Figure 9. NPV profits for revenue linearly increase with number of access in large coverage

\begin{tabular}{|c|c|c|c|c|c|}
\hline \multicolumn{6}{|c|}{ Revenue linearly increase with number of access (Large, small coverage) } \\
\hline revenue per access & 0.0015 & & revenue per access & 0.0015 & \\
\hline SU1 with offloading & NPV-profit & -1337011 & & NPV-profit & 1671 \\
\hline SU2 with offloading & NPV-profit & -3432256 & & NPV-profit & -3613 \\
\hline revenue per access & 0.004 & & revenue per access & 0.004 & \\
\hline SU1 with offloading & NPV-profit & 4079449 & & NPV-profit & 12504 \\
\hline SU2 with offloading & NPV-profit & -1507871 & & NPV-profit & -1587 \\
\hline revenue per access & 0.0015 & & revenue per access & 0.0015 & \\
\hline SU1 without offloading & NPV-profit & 5057640 & & NPV-profit & 5287 \\
\hline SU2 without offloading & NPV-profit & 4489 & & NPV-profit & -3171 \\
\hline \multicolumn{6}{|c|}{ Step revenue function increase with data size (Large, small coverage) } \\
\hline Long holding time with offloading & & & Long holding time with offloading & & \\
\hline SU1 & NPV-profit & -1025379 & & NPV-profit & 2295 \\
\hline SU2 & NPV-profit & 474508 & & NPV-profit & 499 \\
\hline Short holding time with offloading & & & Short holding time with offloading & & \\
\hline SU1 & NPV-profit & -78125.22693 & & NPV-profit & 4189 \\
\hline SU2 & NPV-profit & -242981 & & NPV-profit & -256 \\
\hline Short holding time without offloading & & & Short holding time without offloading & & \\
\hline SU1 & 1 NPV-profit & 4289307 & & NPV-profit & 4410 \\
\hline SU2 & NPV-profit & 444708 & & NPV-profit & 468 \\
\hline \multicolumn{6}{|c|}{ Revenue linearly increase with capacity ( $100 \mathrm{~m}$ radius, $50 \mathrm{~m}$ radius) } \\
\hline ISM-low interference & NPV-profit & 4629 & & NPV-profit & 18513 \\
\hline ISM-high interference & NPV-profit & -4819 & & NPV-profit & -19275 \\
\hline TVWS-low interference & NPV-profit & 2773806 & & NPV-profit & 11087469 \\
\hline TVWS-high interference & NPV-profit & -2049 & & NPV-profit & -8196 \\
\hline
\end{tabular}

Table 3. NPV profits for all cases

From Table 3, it is clear that there are three major categories for revenue function that increases linearly with number of access. In the first case, we test unlicensed users' profits with low unit price while offloading is considered. In the second case, we change the unit price from low to high in order to see the impact from unit price. In the third case, mobile offloading is not considered in order to compare profits that unlicensed users can expect with and without offloading. There are three cases for profits that get from step revenue functions as well. In the first case, we test unlicensed usage with long 
holding time with offloading. In the second case, the holding time change from long to short, in order to compare the impact from holding time. In the third case, mobile offloading is not considered when short holding time is applied. In this way, the impact from mobile offloading is analyzed. In the third section, we analyze profits that unlicensed users can get with combinations of different interference level and frequency bands.

Several observations can be made from Table 3 and Figure 9. First, the unlicensed users' profit is much higher when mobile offloading is not involved. For example, SU1 is profitable even with "L" unit price when there is no mobile offloading. However, the profit switch to negative when the same unit price is applied and mobile offloading is present. SU1 needs to double the unit price to get profits under offloading (which can affect demand). This phenomenon further addresses the risks that are brought by mobile offloading in unlicensed bands. If unlicensed users do not consider mobile offloading when planning the service and confront one when providing service, it may lower their profit and Quality of Services (QoS).

One similar observation can be made from the third part of Table 3. We can see that profits change from positive to negative when interference level increases from low to high. In other words, unlicensed users may lose money if they prepare for low interference and experience higher interference from mobile offloading. It is also clear that with lower frequency (TVWS) and therefore longer propagation distances, unlicensed users can get higher profits under this revenue function.

Second, SU2 is not profitable under both " $L$ " and " $H$ " unit price when revenue increase with number of access linearly. The reason is that the access rate for SU2 is lower than SU1. Therefore, under the same cost model, SU2 is not profitable. It may not be a problem if the infrastructure and maintenance is cheap for SU2.

Third, it is clear that profits are lower in small coverage than in large coverage due to the smaller number of accesses. However, most of the situations in small coverage lead to positive profits. SU1 with lower unit price in the first revenue function can gain profits with small coverage, while unlicensed users lose money when they want to provide large coverage. A similar situation can be found in the second revenue function as well. SU1 with long holding time offloading lead to negative profits when the coverage is large, while unlicensed users can get profits under the same situation by reduce coverage. It is because the population density is high in small coverage compared to large coverage.

Last but not least, although we did not explicitly analyze the impact from operating time, some conclusions can be draw from Table 2 (in Appendix). Not all cases bring profit in the very first year, such as "SU1 without offloading" in large coverage. They need some time to recover from the initial investment. Unlicensed providers can get profits by continuing providing services in these cases. However, in some cases, the longer the operating time, the more unlicensed users lose, such as "SU2 with offloading".

From those observations, here are some suggestions for unlicensed users. First, the unit price needs to be determined based on individual services. It will be impacted by the number of users, the users' data usage, and mobile offloading statistics. One of the reasons that SU2 has less difference between with 
and without mobile offloading is that the SU2 profile has peak usage before dawn, when mobile offloading is low. Therefore, the impact from mobile offloading is low. Thus, in order to prevent high monetary risks, unlicensed users need to consider the distinct impact from mobile offloading. Second, unlicensed users need to be cautious about coverage. Although large coverage can attract more service access and users, it may not necessarily lead to higher profit. There are two reasons for this phenomenon. On one hand, population density is high and the service demand is more certain in smaller coverage. On the other hand, with the increase of coverage, cost for establishing infrastructures, backhaul, and maintenance soar up quickly. Third, although the expected capacity for TVWS is higher than ISM bands, unlicensed users need to consider interference and usage from primary users. Fourth, unlicensed users need to plan operating time with potential service demands and revenues. Longer operating time may leader to higher profits or loss.

\subsection{Mitigation Strategies}

Although we showed that the spectrum that left for unlicensed usage is greatly impacted by incumbents and those impacts may further affect monetary gain for unlicensed users, these risks do not necessarily lead to system failure. Like any engineering investment, unlicensed users have mitigation strategies to cope with risks. Here is a list of mitigations strategies that unlicensed users may have. This is list is by no means exclusive.

- Switch: unlicensed users can switch to other spectrum usage models. For example, they can switch to primary usage when spectrum license is available. They can also opportunistically access the spectrum or lease spectrum and infrastructures from primary users.

- Improve: unlicensed users can improve their services by acquire more spectrum and lease infrastructures.

- Drop: unlicensed users can drop the project if it is not profitable to continue.

Meanwhile, unlicensed usage has its advantages. From the above numerical results we can see that unlicensed usage is flexible, especially with small coverage. Although unlicensed usage may lead to less revenue, the required cost is low as well. Therefore, unlicensed usage is suitable for small corporations to test new services.

\section{Conclusion}

Unlicensed spectrum bands have resulted in a wide range of new wireless devices and services since their introduction. In order to further leverage these advantages, there are numerous advocates favoring an expansion of the quantity of unlicensed spectrum bands. However, increasing the quantity of unlicensed bands may not necessarily bring more unlicensed usage due to the mobile offloading by incumbents. Mobile offloading may deter unlicensed usage in unlicensed bands from two ways. First, as several reports have pointed out, WiFi has already accounted for more than $50 \%$ of mobile data usage. Therefore, the spectrum left for unlicensed usage is lower. Second, due to this reduction in the quantity of spectrum that is left for unlicensed usage and the potential high interference from mobile offloading, unlicensed usage may experience low QoS and higher monetary risks. If this is the case, unlicensed users may not bother to establish infrastructures in the unlicensed bands. 
In this paper, we adopted a time-varying Markov model to estimate the potential usage from mobile offloading. We further analyze monetary risks that unlicensed users may experience by applying three different revenue models. We use these models to compute numerical results for a range of possible operational scenarios.

Several conclusions can be draw from numerical results. First, unlicensed users need to consider mobile offloading during the planning stage, since the profits that they may achieve vary significantly with and without mobile offloading. Second, there is no universal rule for unlicensed users, since mobile offloading traffic varies with services. It is possible that mobile offloading traffic is intense during some period of time, which does not impact unlicensed usage, such as mobile offloading traffic 1 and SU2 shown in Figure 3. Third, coverage and operating time are two other factors that impact unlicensed users' profits as well. Meanwhile, regulators also need to pay attention to mobile offloading in unlicensed bands if the ultimate goal is to stimulate unlicensed usage.

\section{Appendix}

\begin{tabular}{|c|c|c|c|c|c|c|c|c|c|c|c|c|}
\hline & Year & 0 & 1 & 2 & 3 & 4 & 5 & 6 & 7 & 8 & 9 & 10 \\
\hline & cost & -570000 & -617500 & -617500 & -617500 & -617500 & -617500 & -617500 & -617500 & -617500 & -617500 & -617500 \\
\hline revenue per access & 0.0015 & & & & & & & & & & & \\
\hline \multirow[t]{2}{*}{ SU1 with offloading } & revenue cash flow & & 471397.5 & 471397.5 & 471397.5 & 471397.5 & 471397.5 & 471397.5 & 471397.5 & 471397.5 & 471397.5 & 471397.5 \\
\hline & NPV-profit & -570000 & -709012 & -838266 & -955418 & -1058540 & -1146176 & -1217384 & -1271749 & -1309362 & -1330789 & -1337011 \\
\hline \multirow[t]{2}{*}{ SU2 with offloading } & revenue cash flow & & 167480.3 & \begin{tabular}{|l|}
167480.3 \\
\end{tabular} & 167480.3 & 167480.3 & 167480.3 & 167480.3 & 167480.3 & 167480.3 & 167480.3 & 167480.3 \\
\hline & NPV-profit & -570000 & \begin{tabular}{|l|l|}
-1009921 \\
\end{tabular} & \begin{tabular}{|l|}
-1431174 \\
\end{tabular} & -1825868 & -2187083 & -2509113 & -2787637 & -3019821 & -3204340 & -3341326 & -3432256 \\
\hline revenue per access & 0.004 & & & & & & & & & & & \\
\hline \multirow[t]{2}{*}{ SU1 with offloading } & revenue cash flow & & 1257060 & 1257060 & 1257060 & 1257060 & 1257060 & 1257060 & 1257060 & 1257060 & 1257060 & 1257060 \\
\hline & NPV-profit & -570000 & 68871.29 & 694472.4 & 1294799 & 1858881 & 2377181 & 2841906 & 3247226 & 3589382 & 3866690 & 4079449 \\
\hline \multirow[t]{2}{*}{ SU2 with offloading } & revenue cash flow & & 446614 & 446614 & 446614 & 446614 & 446614 & 446614 & 446614 & 446614 & 446614 & 446614 \\
\hline & NPV-profit & -570000 & -733550 & -886616 & -1026401 & -1150569 & -1257319 & -1345434 & -1414299 & -1463892 & -1494742 & -1507871 \\
\hline revenue per access & 0.0015 & & & & & & & & & & & \\
\hline \multirow[t]{2}{*}{ SU1 without offloading } & revenue cash flow & & 733650 & 733650 & 733650 & 733650 & 733650 & 733650 & 733650 & 733650 & 733650 & 733650 \\
\hline & NPV-profit & -570000 & -7236 & 555528 & 1118292 & 1681056 & 2243820 & 2806584 & 3369348 & 3932112 & 4494876 & 5057640 \\
\hline \multirow[t]{2}{*}{ SU2 without offloading } & revenue cash flow & & 228334.9 & 228334.9 & 228334.9 & 228334.9 & 228334.9 & 228334.9 & 228334.9 & 228334.9 & 228334.9 & 228334.9 \\
\hline & NPV-profit & -570000 & -512551 & -455102 & -397653 & -340205 & -282756 & -225307 & -167858 & -110409 & -52960.1 & 4488.75 \\
\hline
\end{tabular}

Table 2. NPV profits for revenue linearly increases with number of access in large coverage

\begin{tabular}{|l|l|r|r|r|r|r|r|r|r|r|r|r|}
\hline & Year & 0 & 1 & 2 & 3 & 4 & 5 & 6 & 7 & 8 & 9 & 10 \\
\hline & cost & -600 & -650 & -650 & -650 & -650 & -650 & -650 & -650 & -650 & -650 & -650 \\
\hline revenue per access & \multicolumn{1}{|c|}{0.0015} & & & & & & & & & & & \\
\hline SU1 with offloading & revenue cash flow & & 942.795 & 942.795 & 942.795 & 942.795 & 942.795 & 942.795 & 942.795 & 942.795 & 942.795 & 942.795 \\
\hline & NPV-profit & -600 & -304.163 & -11.1444 & 273.3673 & 544.0709 & 796.2492 & 1025.929 & 1229.998 & 1406.273 & 1553.526 & 1671.45 \\
\hline SU2 with offloading & revenue cash flow & & 176.295 & 176.295 & 176.295 & 176.295 & 176.295 & 176.295 & 176.295 & 176.295 & 176.295 & 176.295 \\
\hline & NPV-profit & -600 & -1063.07 & -1506.5 & -1921.97 & -2302.19 & -2641.17 & -2934.35 & -3178.76 & -3372.99 & -3517.19 & -3612.9 \\
\hline revenue per access & \multicolumn{1}{|c|}{0.004} & & & & & & & & & & & \\
\hline SU1 with offloading & revenue cash flow & & 2514.12 & 2514.12 & 2514.12 & 2514.12 & 2514.12 & 2514.12 & 2514.12 & 2514.12 & 2514.12 & 2514.12 \\
\hline & NPV-profit & -600 & 1251.604 & 3054.332 & 4773.801 & 6378.912 & 7842.962 & 9144.51 & 10267.95 & 11203.76 & 11948.48 & 12504.37 \\
\hline SU2 with offloading & revenue cash flow & & 470.12 & 470.12 & 470.12 & 470.12 & 470.12 & 470.12 & 470.12 & 470.12 & 470.12 & 470.12 \\
\hline & NPV-profit & -600 & -772.158 & -933.279 & -1080.42 & -1211.13 & -1323.49 & -1416.25 & -1488.74 & -1540.94 & -1573.41 & -1587.23 \\
\hline revenue per access & \multicolumn{1}{|c|}{0.0015} & & & & & & & & & & & \\
\hline SU1 without offloading & revenue cash flow & & 1467.3 & 1467.3 & 1467.3 & 1467.3 & 1467.3 & 1467.3 & 1467.3 & 1467.3 & 1467.3 & 1467.3 \\
\hline & NPV-profit & -600 & 215.1485 & 1012.105 & 1775.603 & 2491.728 & 3148.427 & 3735.894 & 4246.847 & 4676.654 & 5023.341 & 5287.456 \\
\hline SU2 without offloading & revenue cash flow & & 240.3525 & 240.3525 & 240.3525 & 240.3525 & 240.3525 & 240.3525 & 240.3525 & 240.3525 & 240.3525 & 240.3525 \\
\hline & NPV-profit & -600 & -999.651 & -1381.53 & -1738.5 & -2064.33 & -2353.9 & -2603.39 & -2810.31 & -2973.58 & -3093.42 & -3171.28 \\
\hline
\end{tabular}

Table 3. NPV profits for revenue linearly increases with number of access in small coverage 


\begin{tabular}{|c|c|c|c|c|c|c|c|c|c|c|c|c|}
\hline & Year & 0 & 1 & 2 & 3 & 4 & 5 & 6 & 7 & 8 & 9 & 10 \\
\hline & cost & -570000 & -617500 & -617500 & -617500 & -617500 & -617500 & -617500 & -617500 & -617500 & -617500 & -617500 \\
\hline \multirow[t]{2}{*}{ SU1 long holding time with offloading } & revenue cash flow & & 516600 & 516600 & 516600 & 516600 & 516600 & 516600 & 516600 & 516600 & 516600 & 516600 \\
\hline & NPV-profit & -570000 & -664257 & -750081 & -825954 & -890688 & -943462 & -983836 & -1011753 & -1027516 & -1031756 & -1025379 \\
\hline \multirow[t]{2}{*}{ SU1 short holding time with offloading } & revenue cash flow & & 654000 & 654000 & 654000 & 654000 & 654000 & 654000 & 654000 & 654000 & 654000 & 654000 \\
\hline & NPV-profit & -570000 & -528218 & -482029 & -432426 & -380477 & -327282 & -273930 & -221455 & -170803 & -122799 & \begin{tabular}{|l|}
-78125.2 \\
\end{tabular} \\
\hline \multirow[t]{2}{*}{ SU2 long holding time with offloading } & revenue cash flow & & 734160 & 734160 & 734160 & 734160 & 734160 & 734160 & 734160 & 734160 & 734160 & 734160 \\
\hline & NPV-profit & -570000 & -448851 & -325646 & -202840 & -82817 & 32200.41 & 140233.2 & 239609 & 329008.4 & \begin{tabular}{|l|}
407492.4 \\
\end{tabular} & 474508.3 \\
\hline \multirow[t]{2}{*}{ SU2 short holding time with offloading } & revenue cash flow & & 630087.5 & 630087.5 & 630087.5 & 630087.5 & 630087.5 & 630087.5 & 630087.5 & 630087.5 & 630087.5 & 630087.5 \\
\hline & NPV-profit & -570000 & -551894 & -528680 & -500914 & \begin{tabular}{|l|}
-469272 \\
\end{tabular} & -434520 & -397479 & -358995 & \begin{tabular}{|l|}
-319902 \\
\end{tabular} & -280990 & -242981 \\
\hline \multirow{2}{*}{\begin{tabular}{|l|} 
SU1 short holding time without offloading \\
\end{tabular}} & revenue cash flow & & 670000 & 670000 & 670000 & 670000 & 670000 & 670000 & 670000 & 670000 & 670000 & 670000 \\
\hline & NPV-profit & -570000 & 99009.9 & 753857.4 & 1381982 & 1971914 & 2513691 & 2999181 & 3422311 & 3779181 & 4068063 & 4289307 \\
\hline \multirow{2}{*}{ SU2 short holding time without offloading } & revenue cash flow & & 729837.5 & 729837.5 & 729837.5 & 729837.5 & 729837.5 & 729837.5 & 729837.5 & 729837.5 & 729837.5 & 729837.5 \\
\hline & NPV-profit & -570000 & -453131 & -334079 & -215220 & -98867.9 & 12815.87 & 117900.1 & 214746.8 & 302056.9 & 378897.3 & 444708.4 \\
\hline
\end{tabular}

Table 4. NPV profits for stepwise revenue function with data size in large coverage

\begin{tabular}{|c|c|c|c|c|c|c|c|c|c|c|c|c|}
\hline & Year & 0 & 1 & 2 & 3 & 4 & 5 & 6 & 7 & 8 & 9 & 10 \\
\hline & cost & -600 & -650 & -650 & -650 & -650 & -650 & -650 & -650 & -650 & -650 & -650 \\
\hline \multirow{2}{*}{ SU 1 long holding time with offloading } & revenue cash flow & & 1033.2 & 1033.2 & 1033.2 & 1033.2 & 1033.2 & 1033.2 & 1033.2 & 1033.2 & 1033.2 & 1033.2 \\
\hline & NPV-profit & -600 & -214.653 & 165.2255 & 532.2964 & 879.774 & 1201.676 & 1493.025 & 1749.989 & 1969.964 & 2151.592 & 2294.714 \\
\hline \multirow[t]{2}{*}{ SU1 short holding time with offloading } & revenue cash flow & & 1308 & 1308 & 1308 & 1308 & 1308 & 1308 & 1308 & 1308 & 1308 & 1308 \\
\hline & NPV-profit & -600 & 57.42574 & 701.329 & 1319.351 & 1900.196 & 2434.036 & 2912.837 & 3330.584 & 3683.391 & 3969.507 & 4189.221 \\
\hline \multirow[t]{2}{*}{ SU2 long holding time with offloading } & revenue cash flow & & 772.8 & 772.8 & 772.8 & 772.8 & 772.8 & 772.8 & 772.8 & 772.8 & 772.8 & 772.8 \\
\hline & NPV-profit & -600 & -472.475 & -342.785 & -213.516 & -87.1758 & 33.89516 & 147.6139 & 252.22 & 346.3246 & 428.9393 & 499.4824 \\
\hline \multirow[t]{2}{*}{ SU2 short holding time with offloading } & revenue cash flow & & 663.25 & 663.25 & 663.25 & 663.25 & 663.25 & 663.25 & 663.25 & 663.25 & 663.25 & 663.25 \\
\hline & NPV-profit & -600 & -580.941 & -556.505 & -527.278 & -493.971 & -457.389 & -418.399 & -377.89 & -336.739 & -295.779 & -255.77 \\
\hline \multirow[t]{2}{*}{ SU1 short holding time without offloading } & revenue cash flow & & 1340 & 1340 & 1340 & 1340 & 1340 & 1340 & 1340 & 1340 & 1340 & 1340 \\
\hline & NPV-profit & -600 & 89.10891 & 763.7574 & 1411.003 & 2019.022 & 2577.542 & 3078.172 & 3514.642 & 3882.916 & 4181.2 & 4409.833 \\
\hline \multirow[t]{2}{*}{ SU2 short holding time without offloading } & revenue cash flow & & 768.25 & 768.25 & 768.25 & 768.25 & 768.25 & 768.25 & 768.25 & 768.25 & 768.25 & 768.25 \\
\hline & NPV-profit & -600 & -476.98 & -351.662 & -226.547 & -104.071 & 13.49038 & 124.1054 & 226.0493 & 317.9546 & 398.8392 & 468.1141 \\
\hline
\end{tabular}

Table 5. NPV profits for stepwise revenue function with data size in small coverage

\begin{tabular}{|c|c|c|c|c|c|c|c|c|c|c|c|c|}
\hline & Year & 0 & 1 & 2 & 3 & 4 & 5 & 6 & 7 & 8 & 9 & 10 \\
\hline & $\cos t$ & -600 & -650 & -650 & -650 & -650 & -650 & -650 & -650 & -650 & -650 & -650 \\
\hline \multirow[t]{2}{*}{ ISM-low interference } & revenue cash flow & & 1371.759 & 1371.759 & 1371.759 & 1371.759 & 1371.759 & 1371.759 & 1371.759 & 1371.759 & 1371.759 & 1371.759 \\
\hline & NPV-profit & -600 & 120.5538 & 825.7162 & 1501.964 & 2136.955 & 2719.968 & 3242.263 & 3697.315 & 4080.941 & 4391.3 & 4628.786 \\
\hline \multirow[t]{2}{*}{ ISM-high interference } & revenue cash flow & & 1.371786 & 1.371786 & 1.371786 & 1.371786 & 1.371786 & 1.371786 & 1.371786 & 1.371786 & 1.371786 & 1.371786 \\
\hline & NPV-profit & -600 & -1236.27 & -1847.75 & -2422.96 & -2951.74 & -3425.63 & -3838.13 & -4184.88 & -4463.67 & -4674.37 & -4818.84 \\
\hline \multirow[t]{2}{*}{ TVWS-low interference } & revenue cash flow & & 403043.4 & 403043.4 & 403043.4 & 403043.4 & 403043.4 & 403043.4 & 403043.4 & 403043.4 & 403043.4 & 403043.4 \\
\hline & NPV-profit & -600 & 397815.3 & 784441.4 & 1151930 & 1493674 & 1804043 & 2078563 & 2314033 & 2508574 & 2661614 & 2773806 \\
\hline \multirow[t]{2}{*}{ TVWS-high interference } & revenue cash flow & & 403.1372 & 403.1372 & 403.1372 & 403.1372 & 403.1372 & 403.1372 & 403.1372 & 403.1372 & 403.1372 & 403.1372 \\
\hline & NPV-profit & -600 & -838.478 & -1063.96 & -1272.27 & -1459.85 & -1623.88 & -1762.33 & -1874.01 & -1958.59 & -2016.53 & -2049.02 \\
\hline
\end{tabular}

Table 6. NPV profits for revenue increases linearly with capacity (100m radius)

\begin{tabular}{|c|c|c|c|c|c|c|c|c|c|c|c|c|}
\hline & Year & 0 & 1 & 2 & 3 & 4 & 5 & 6 & 7 & 8 & 9 & 10 \\
\hline & cost & -2400 & -2600 & -2600 & -2600 & -2600 & -2600 & -2600 & -2600 & -2600 & -2600 & -2600 \\
\hline \multirow[t]{2}{*}{ ISM-low interference } & revenue cash flow & & 5486.711 & 5486.711 & 5486.711 & 5486.711 & 5486.711 & 5486.711 & 5486.711 & 5486.711 & 5486.711 & 5486.711 \\
\hline & NPV-profit & -2400 & 481.8923 & 3302.229 & 6006.924 & 8546.609 & 10878.41 & 12967.37 & 14787.39 & 16321.73 & 17563.04 & 18512.9 \\
\hline \multirow[t]{2}{*}{ ISM-high interference } & revenue cash flow & & 5.487145 & 5.487145 & 5.487145 & 5.487145 & 5.487145 & 5.487145 & 5.487145 & 5.487145 & 5.487145 & 5.487145 \\
\hline & $N P V-p$ & -2400 & -4945.06 & -7391.02 & -9691.86 & -11807 & -13702.5 & -15352.5 & -16739.5 & -17854.7 & -18697.5 & -19275.4 \\
\hline \multirow[t]{2}{*}{ TVWS-low interference } & reve & & 1611049 & 1611049 & 1611049 & 1611049 & 1611049 & 1611049 & 1611049 & 1611049 & 1611049 & 1611049 \\
\hline & NPV-p & -2400 & 1590147 & 3135571 & 4604499 & 5970521 & 7211130 & 8308442 & 9249663 & 10027284 & 10639014 & 11087469 \\
\hline \multirow[t]{2}{*}{ TVWS-high interference } & revent & & 1612.548 & 1612.548 & 1612.548 & 1612.548 & 1612.548 & 1612.548 & 1612.548 & 1612.548 & 1612.548 & 1612.548 \\
\hline & NPV-profit & -2400 & $\mid-3353.91$ & -4255.82 & \begin{tabular}{|l|}
-5089.07 \\
\end{tabular} & -5839.42 & $\mid-6495.54$ & -7049.31 & -7496.04 & $\mid-7834.36$ & \begin{tabular}{|l|}
-8066.13 \\
\end{tabular} & -8196.09 \\
\hline
\end{tabular}

Table 7. NPV profits for revenue increases linearly with capacity (50m radius) 


\section{Reference}

Dimatteo, S., Hui, P., Han, B., \& Li, V. O. (2011, October). Cellular traffic offloading through WiFi networks. In Mobile Adhoc and Sensor Systems (MASS), 2011 IEEE 8th International Conference on (pp. 192-201). IEEE.

Dudda, T., \& Irnich, T. (2012, October). Capacity of cellular networks deployed in TV White Space. In Dynamic Spectrum Access Networks (DYSPAN), 2012 IEEE International Symposium on (pp. 254-265). IEEE.

FCC, F. (2008). FCC 08-260. Unlicensed Operation in the TV Broadcast Bands. Available: http://hraunfoss.fcc.gov/edocs_public/attachmatch/FCC-08-260A1.pdf

FCC. (2010). Second Memorandum Opinion and Order (FCC 10-174). US Govt. Printing Office, Washington, DC, 23. Available: http://hraunfoss.fcc.gov/edocs_public/attachmatch/FCC-10-174A1.pdf FCC. (2912). Third Memorandum Opinion and Order (FCC 12-36). Available: http://hraunfoss.fcc.gov/edocs_public/attachmatch/FCC-12-36A1.pdf

GrøNsund, P., GrøNdalen, O., \& LäHteenoja, M. (2012). Business case evaluations for LTE network offloading with cognitive femtocells.Telecommunications Policy.

Han, B., Hui, P., \& Srinivasan, A. (2011). Mobile data offloading in metropolitan area networks. ACM SIGMOBILE Mobile Computing and Communications Review, 14(4), 28-30.

Harrison, K., Mishra, S. M., \& Sahai, A. (2010, April). How much white-space capacity is there?. In New Frontiers in Dynamic Spectrum, 2010 IEEE Symposium on (pp. 1-10). IEEE.

Hu, L., Coletti, C., Huan, N., Mogensen, P., \& Elling, J. (2012, May). How Much Can Wi-Fi Offload? A Large-Scale Dense-Urban Indoor Deployment Study. In Vehicular Technology Conference (VTC Spring), 2012 IEEE 75th (pp. 1-6). IEEE.

Hui, P., Crowcroft, J., \& Yoneki, E. (2011). Bubble rap: Social-based forwarding in delay-tolerant networks. Mobile Computing, IEEE Transactions on, 10(11), 1576-1589.

Informa, (2013) Understanding today's smartphone user-an updated and expanded analysis of datausage patterns in six of the worlds' most advanced 4F LTE market. Available:

http://www.mobidia.com/admin/whitepaper/6.pdf?utm_source=Facebook\&utm_medium=Tangence\&u tm_campaign=Social+Media

Lee, K., Lee, J., Yi, Y., Rhee, I., \& Chong, S. (2010, November). Mobile data offloading: how much can WiFi deliver?. In Proceedings of the 6th International COnference (p. 26). ACM.

Mishra, S., \& Sahai, A. (2010). How much white space has the fcc opened up.IEEE Communication Letters. 
Musey, A. (2013) Could incumbents wireless companies also dominate unlicensed spectrum? Available: http://summitridgegroup.com/could-incumbents-wireless-companies-also-dominate-unlicensedspectrum/

Nekovee, M. (2010, April). Cognitive radio access to TV white spaces: Spectrum opportunities, commercial applications and remaining technology challenges. In New Frontiers in Dynamic Spectrum, 2010 IEEE Symposium on(pp. 1-10). IEEE.

Patil, K., Deshmukh, M., \& Cornean, H. (2010, June). CTMC based spectrum sharing scheme for cognitive radio networks. In Communications (COMM), 2010 8th International Conference on (pp. 509-512). IEEE.

Simsek, M., Bennis, M., Debbah, M., \& Czylwik, A. (2012) Rethinking Offload: How to Intelligently Combine Wi-Fi and Small Cells?.

Snider, J. H. (2007). The Art of Spectrum Lobbying: America's $\$ 480$ Billion Spectrum Giveaway, How it Happened, and How to Prevent it from Recurring.New America Foundation, Tech. Rep., Aug.

Tipper, D., \& Sundareshan, M. K. (1990). Numerical methods for modeling computer networks under nonstationary conditions. Selected Areas in Communications, IEEE Journal on, 8(9), 1682-1695.

Van De Beek, J., Riihijarvi, J., Achtzehn, A., \& Mahonen, P. (2011, May). UHF white space in Europe-a quantitative study into the potential of the $470-790 \mathrm{MHz}$ band. In New Frontiers in Dynamic Spectrum Access Networks (DySPAN), 2011 IEEE Symposium on (pp. 1-9). IEEE.

Willkomm, D., Machiraju, S., Bolot, J., \& Wolisz, A. (2008, October). Primary users in cellular networks: A large-scale measurement study. In New frontiers in dynamic spectrum access networks, 2008. DySPAN 2008. 3rd IEEE symposium on (pp. 1-11). IEEE. 